\title{
The Empirical Analysis of Effect of Origin of Alma Mater on University Teachers Interschool Mobility ${ }^{*}$
}

\author{
Peiqun Wu, Cui Meng \\ Department of foundation, Beijing Electronic Science and Technology Institute, Fengtai, Beijing, China \\ pqw66@sina.com
}

\begin{abstract}
We get a representative large sample including 4,890 teachers from 27 universities, the interschool mobile data of Chinese university teachers involving 2,354 effective resumes are collected from corresponding university's official website. By using the index of "per capita career mobile expectation" and "relationship between the alma mater and the current school" and the method of the data statistical analysis, We establish the model on impact factor and function of Chinese university teachers interschool mobility. Results of analysis show that, the high ratio of teachers graduated from the same university is one cause of university teacher interschool too low mobility, the origin of alma mater becomes more closer, the teacher reaches the university by flowing more easily.
\end{abstract}

Index Terms - origin of alma mater, interschool mobility, correlation analysis, regression analysis, university system

The origin of alma mater, refers to the origin relationship between students and academic factions in the education and scientific research. This paper refers specifically to the relationship between the university teacher's alma mater and his serving university. University teacher's interschool mobility refers to the change situation of teachers in full-time universities (except part-time). What is the structure of university teachers' origin of alma mater in China? What's its role in the university teachers' interschool mobility and their academic status? Empirical research in the problem could provide an objective basis necessary for deepening the reform of university personnel system and establishing of the modern university system in China

\section{Review of Existing Relevant Research}

By doing empirical analyses of typical universities or universities that the typical subject teachers graduated from, Some Chinese scholars revealed the "inbreeding" seriousness of academic structure in civil universities, especially research -oriented universities (Yuan Tian, Kai Yu, 2013; Bin Zhang, 2013). [1-2] Empirical studies on a large sample of university teachers also reveals the relation between the high rate that university teachers graduated from the same university and low mobility (Peiqun Wu, 2011; Peiqun Wu, Huamin Feng, 2013). [3-4] By doing empirical analyses of American university teachers in the last century, We find the fact that effect of university that the teacher graduated from is greater than the effect of teachers' academic performance in employment of the teacher, University's allocation of talent is decided by "belonged process"( Crane,D.,1965,1970; Long, J.S.\& Allison, P.D.,1962,1963; Reskin,B.F.,1979). Japanese education sociologist Atsunori Yamanoi (1990, 2005). Integrated content analysis with survey methods to obtain relevant data of Japan university teachers, statistical analysis of these data confirmed the role of origin of alma mater of Japanese university teachers in rising mobility, That is "selection configuration by the control of the alma mater clique "mechanism of teachers' mobility. [5-6]

What is the role of origin of alma mater to university teachers' interschool mobility? Based on the author's own large sample data, we do statistical analysis and make accordingly discussion.

\section{Source of Data and Analytic Index}

\subsection{Source of Data: the self-built database by Web content analytic method}

Firstly, we collect stratified cluster sample of civil university teachers according to the order of Region Province - University - College - Department to determine a sample of 4,890 teachers from 27 public universities ; for each teacher in the sample, teacher 's introduction on the official website of the university as the main source of information, We collect their resume data by the end of 2008 and analyze content of the information, finally, We get database including the basic information of 2,354 teachers and school professional experience information (referred to as "effective sample data").[7]

\subsection{Analysis index and its calculation method}

\subsubsection{Mobility index: per capita career mobile expectation}

Interschool mobility, refers to how often the teachers Interschool mobile, it could by described by "mobile frequency" and "the per capita number of flows ", however, greatly influenced by sample age factor, the two indexes are not conducive to horizontal comparison. "Per capita career mobility expectation value " (with "E"), can eliminate index of age factor. The formula for calculating the index is as follows. [8]

$$
E=\frac{1}{N} \sum_{i}\left(\frac{f_{i}}{m_{i}} \cdot M_{i}\right)
$$

$f_{i}$ and $^{m_{i}}$ mean interschool mobile times and university's

\footnotetext{
* This work is supported by Human and Social Science Funding Project granted by the Ministry of Education \#12YJA880124, Higher Education Reform Project granted by Beijing \#2013Joint03, the Fundamental Research funds for the Central Universities \#2014SK03.
} 
teaching age of the i-th teacher in the effective sample, $M_{i}$ means the expectation of the teacher's total university teaching age. Atsunori Yamanoi (1996) and Peiqun Wu (2011) in their statistical analysis regarded $M_{i}$ as 30 years, so the above equation becomes:

$$
E=\frac{1}{N} \sum_{i}\left(\frac{f_{i}}{m_{i}} \cdot 30\right)
$$

2.2.2 Quantitative indexes of academic status, origin of alma mater and other variables

The main harvest of university teachers by interschool flowing is the academic level that regarded as one of the signs of academic position in new school, so we regard the academic level as variables, "1", "2", "3" respectively represents research universities, teaching and research universities and teaching university, the greater the value becomes, the lower the academic level means. We value other variables, such as origin of alma mater, teacher's academic degree and professional title shown in Table 1. "time of school " and " age of school " respectively refers to the current time and age that the teacher works in the current university. According to the relationship between alma mater and current university: "origin of alma mater" of teachers is given a number shown as follows:

"1": graduated from the currently vocational school only;

"2": the highest degree obtained in the currently vocational school, degree obtained in other schools;

"3": the highest degree obtained in other schools, degree obtained the currently vocational school;

"4": No degree of the currently vocational school.

TABLE 1 Value of Variable

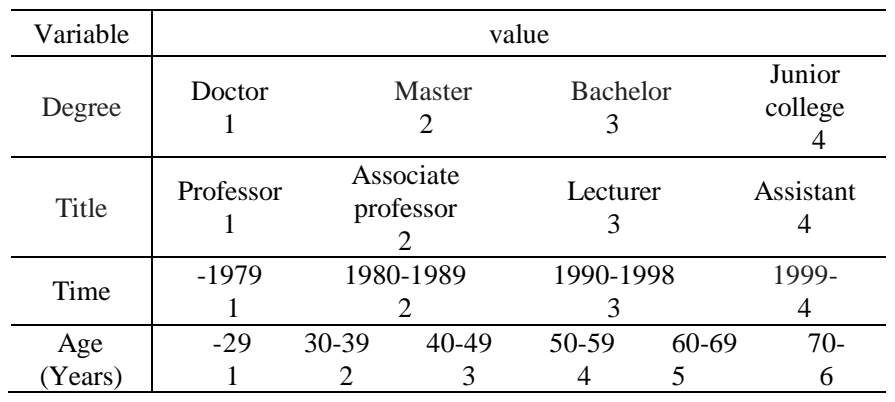

\subsection{Tools of statistical analysis}

Our statistical analysis and modeling are mainly done with the software package SPSS17.0.

\section{Statistical Results and Analysis}

3.1 The structure of origin of alma mater of university teachers

By calculating the value of origin of alma mater on each case in the effective sample, we obtain the frequency distribution of origin of alma mater as shown in Table 2.
TABLE 2 Composition of Alma Mater

\begin{tabular}{|c|c|c|c|c|}
\hline origin of alma mater & 1 & 2 & 3 & 4 \\
\hline $\begin{array}{c}\text { frequency } \\
\text { (percentage\%) }\end{array}$ & $925(41.4)$ & $252(11.3)$ & $111(5.0)$ & $945(42.3)$ \\
\hline
\end{tabular}

We can see from Table 2 that the rate of university teachers that graduated from the school in China averaged $57.7 \%$, even apart from the teachers who even learned in the other schools, the "pure" rate is more than $40 \%$. Statistics on structure of origin of alma mater in some research universities shows that their rates are up to $70 \%$, still much higher than the rate of research universities in Japan.

\subsection{Effects of origin of alma mater on interschool mobility of university teachers}

By grouping the sample according to the value of origin of alma mater, we use the formula (2) to calculate" average career mobility expectation" (signed as" Expectation(E) ") and "number of effective cases " (signed as" Number(N) ") for each group, they are shown as Table 3.

TABLE 3 Mobility of Alma mater for each group

\begin{tabular}{|c|c|c|c|}
\hline Origin of alma mater & Expectation (E) & Number $(\mathrm{N})$ & Standard deviation \\
\hline 1 & 0.03 & 892 & .280 \\
\hline 2 & 0.44 & 215 & .893 \\
\hline 3 & 0.87 & 104 & 2.205 \\
\hline 4 & 0.63 & 897 & 1.275 \\
\hline total & 0.37 & 2108 & 1.065 \\
\hline
\end{tabular}

Values of the four groups are: $0.03,0.44,0.87$ and 0.63 , And the difference between groups was significant, especially $\mathrm{E}$ values of the first group are much lower than the other groups, i.e. teachers who have no external school experience mostly don't want Interschool mobile; The few teachers who graduated from current school but obtained the highest degree in other school (Group Third) have the highest Interschool mobility, i.e. if he left the original "academic circles", it is the most difficult to "return to the origin school", And the mobile type of "U" shaped are more common between the university teachers in Japan, extensity of teachers' origin of alma mater is encouraged in Europe and America. There are difference between China and foreign countries.

Different values of origin of alma mater produce significantly difference between the corresponding mobility of each group, it means that origin of alma mater is an important factor which has a significant effect on the interschool mobility. Then we get the following conclusion:

Conclusion 1: origin of alma mater has a significant impact on interschool mobility of university teachers. the interschool mobility of the teachers who haven't the experience in the other schools is significantly lower than the teachers who have. In the selection of the teachers, university firstly choose the graduate from the same school. 


\subsection{The role of origin of alma mater in achievement of the} teacher's academic status

3.3.1What are the personal factors that are significantly associated with achievement of the teacher's academic status ?

In this paper, there are 395 teachers who have interschool mobile experience in valid samples; the 395 teachers form the "mobile samples". Firstly according to the method shown in Table 1, we quantify the seven major factors (variables) as follows, their current academic level, origin of alma mater, education degree, academic level in the first vocational school, the time of going into the school, title and age, we calculate the correlation coefficient between these variables, obtained data is shown in Table 4. We define the mathematical symbols as follows in Table 4, y means "academic level in currently vocational school", $x_{1}$ means "origin of alma mater", $x_{2}$ means "degree before going into the current school", $x_{3}$ means " academic level in the first vocational school", $x_{4}$ means "the number of new degree from participating in the work to going into the current school", $x_{5}$ means "the time of going into the school", $x_{6}$ means "title", $x_{7}$ means "the age of going into the school".

TABLE 4 Kendall's Correlation Coefficient Table

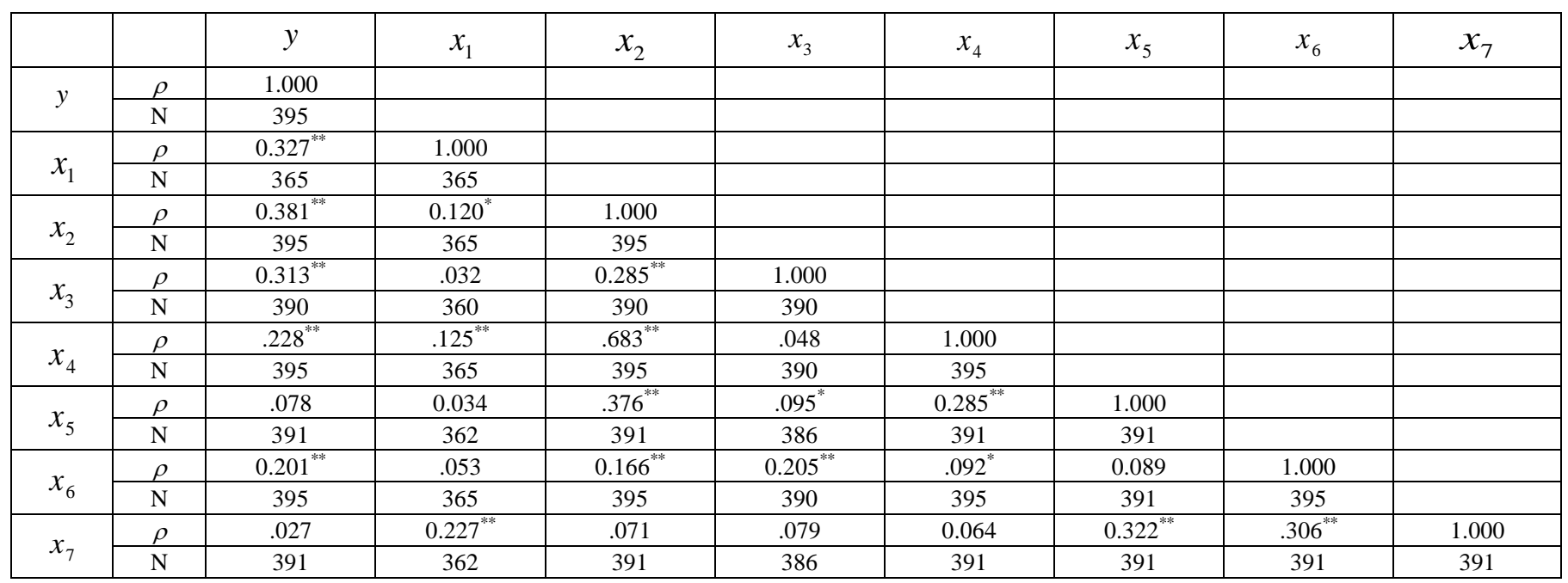

Note: $\rho$ is the correlation coefficient; $\mathrm{N}$ is the effective number of cases; tables for each correlation coefficient is Kendall's correlation coefficient; "**" indicates that at 0.01 significance level was significantly related, "*" indicates the 0.05 significance level was significantly related.

As can be seen, "time of going into the school" and "age of going into the school" aren't significantly related with teachers' current school, but these are related with the "new degree number", "degree before going into the current school", ,"title "and "origin of alma mater" and so on factors.

Arranged in descending order of the correlation coefficient, significant factors related to "academic level in currently vocational school" are shown as follows : "degree before going into the current school", "origin of alma mater", " academic level in the first vocational school", "the number of new degree from participating in the work to going into the current school" and "title", the correlation coefficient between "the number of new degree" and "academic level in currently vocational school" is negative, the correlation coefficient between "degree before going into the current school" and "academic level in currently vocational school" (0.381) is greater than the correlation coefficient between "origin of alma mater" and "academic level in currently vocational school" (0.327)".

However, this is a general correlation coefficient, other factors aren't controlled, by controlling other factors, we could get "net correlation coefficient", we get "net correlation coefficients" between "origin of alma mater" or "degree before going into the current school" and "academic level in currently vocational school" ( 0.409 and 0.360$)$ by controlling the factors of "profession", " title", " academic level in the first vocational school" and "the time of going into the school", the former is higher than the latter, it can be concluded that, academic status of university teachers obtained by interschool mobility is closely related to "origin of alma mater".

\subsubsection{Attribution teachers get academic status}

Further to study the role of the origin of alma mater and the degree of teachers to academic level, we need to do regression analysis of the above factors.

We can select university teachers four factors of "academic degree 1 "university one is graduated from", "the first vocational school "and "title" as independent variables, academic level of university teachers at current school as the dependent variable, we get the general regression equation and standardized regression equation shown as follows by doing linear regression: ${ }^{\mathbb{1}}$

\footnotetext{
${ }^{\infty}$ For simplicity's sake, the right of the regression equation residuals none marked.
} 


$$
\begin{aligned}
& y=0.302 x_{1}+0.260 x_{2}+0.200 x_{3}+0.175 x_{6}-0.574 \\
& y=0.334 x_{1}+0.292 x_{2}+0.251 x_{3}+0.133 x_{6}
\end{aligned}
$$

The coefficient of determination of equation (3) and (4) is 0.357; the two equations can explain $35.7 \%$ difference between the current academic statuses of teachers of the universities in China. In addition, equation (3) indicates that other factors in the same situation, each increase of one degree level qualifications, such as increased by the master's degree to doctorate level ,the level of the university will be improved 0.260 level; The role of the other three factors on the obtaining of academic status could be also explained.

Standardized regression coefficients of the four factors in equation (4) are respectively $0.292,0.334,0.251$ and 0.133 ,it tells us that the role of origin of alma mater in flowing into the higher level university is the largest, in order ,followed by academic degrees, the first vocational school, and the role of the title is the smallest of the four factors.

Conclusion 2: the factors of teachers that play a major role in obtaining the academic status by flowing are mainly origin of alma mater, degree, academic level in former school and title. The role of origin of alma mater is the largest, in order, degree, academic level in former school, the smallest is the title of the teachers.

\section{Further Discussions}

The four factors of university teachers, origin of alma mater, academic degree, professional title and former schools belong to the academic factors of teachers. Our model of the paper pointed out that the four teachers' personal academic factors could determine more than a third of the difference between academic status. Compared with individual family background and other social capital, individual academic factors can account for such a large proportion in the academic status, it shows the advantage of teachers' society in China. However, the origin of alma mater is different from the academic degree, the former vocational school and titles, "academic degree" and "title" can be seen as the academic level of individual teachers, we call these as "self nature", the "origin of alma mater " and "former vocational school" are characterized by groups of teachers, we call these as "group nature", the role of "self nature" corresponds to "performance" or "universalism", shown as "the ability flow"; role of "group nature" corresponds to the "specialism", the corresponding flow more appear as "asylum flow".

In this paper, the model shows that the role of "group nature" for teachers in getting academic status by flowing is greater than "self nature". We can say that the flow of teachers not only follow the universalism but also follow the "specialism", but apparently the "specialism" is higher priority stressed than universalism.

Interschool mobility of university teachers is "the ability flow" under the protection of the origin of alma mater.

The origin of alma mater once identified, it can't be changed, like the human blood relationship. it has no ground for blame that origin of alma mater for university teachers plays the role in getting status, But the role is significantly higher than academic degree and title, It is very easy to cause the case common occur: The university teacher who graduated from the school that distantly related to current school in origin of alma mater, no matter how try hard or academic success, it is difficult to reach his expected status. This is clearly contrary to the modern social fairness, but also difficult to stimulate the development of academic. Our universities should make efforts to overcome this problem in the employment of teachers and school talent exchange, we should further deepen the reform of appointment system and establish the modern university system.

\section{References}

[1] Yuan Tian, Kai Yu, "Models of Faculty in Asia's World-Class Universities", Fudan Education Forum, vol. 11, no.1, pp. 23-49, January 2013.

[2] Bin Zhang, "Departmental Stratification and Its Hierarchical Characteristics in the Ph.D. Exchange Network-A Social Network Analysis Based on Academic Structure of Part of Physics Scholars", Education Research, No. 1, pp. 84-96, January 2013.

[3] [7][8] Peiqun Wu, Chinese-Japanese Comparative Study on Academics profession Mobility, Beijing: Xiyuan Publishing House, pp.49-101. 2011.

[4] Peiqun Wu, Huamin Feng, The interschool mobility of academic profession--- A comparison between web - based content analysis data and international investigation. Science Research Management. Vol.34, No.5, pp.86-92, May 2013.

[5] Atsunori Yamanoi, Study of Social Mobility of College Professors. Tokyo, Japan: Toshindou, pp.24-30, 1990.

[6] Atsunori Yamanoi, Again Study of Japanese academic professors marketplace. COE Research Series 15, Hiroshima, Japan: Hiroshima University Research Institute for Higher Education, pp.1-37, 2005. 